\title{
Vitamin E as a Functional and Biocompatibility Modifier of Synthetic Hemodialyzer Membranes: An Overview of the Literature on Vitamin E-Modified Hemodialyzer Membranes
}

\author{
Marta Piroddi ${ }^{a}$ Francesca Pilolli $^{a}$ Masaharu Aritomi ${ }^{b}$ Francesco Galli ${ }^{a}$ \\ ${ }^{a}$ Department of Internal Medicine, University of Perugia, Perugia, Italy; ${ }^{b}$ Asahi Kasei Medical Co. Ltd, \\ Tokyo, Japan
}

\author{
Key Words \\ Vitamin E · Hemodialysis membranes • Biocompatibility • \\ Oxidative stress $\cdot$ Antioxidants $\cdot$ Inflammation $\cdot$ Uremic \\ anemia
}

\begin{abstract}
Along with one century of history, research has provided many solutions for hemodialysis (HD) biomaterials, encompassing several generations of copolymers that have found wide application in the development of hollow-fiber dialyzer membranes. Polysulfone-based biomaterials have gained increasing consideration and are now the gold standard in the production of biocompatible hemodialyzers. However, even the highest biocompatibility now available cannot exclude that dialyzer membranes and the overall extracorporeal circulation may produce at the subclinical level immunoinflammatory reactions and thus an increased cardiovascular risk of patients on regular HD therapy. The lipophilic antioxidant and radical scavenger vitamin $\mathrm{E}$ has been used (as $\alpha$-tocopherol) to modify cellulosic and synthetic hollowfiber membranes with the ultimate goal to neutralize harmful reactive species and to mimic lipid structures of blood cell plasmalemma and lipoprotein particles. Besides filtration and biocompatibility, this modifier has introduced a third
\end{abstract}

function of dialyzer membranes, namely 'antioxidant bioactivity'. Vitamin E can also serve as a template molecule to produce synthetic redox-active and -silent (non-antioxidant) modifiers for future generations of dialyzer membranes. This mini-review article describes the evolution of vitamin E-derived copolymers as a generation of biomaterials that has offered a clinical challenge and still represents a chance to further improving the quality of HD therapy.

Copyright $\odot 2012$ S. Karger AG, Basel

\section{Introduction: The Road from Biocompatibility to Bioactivity}

The concept of biocompatibility with its different definitions remains central to the effort of the biomaterial industry and clinicians to provide high-quality hemodialysis (HD) therapies. Operational aspects may lead to consider biomaterials as 'inert surfaces' that implies 'the lack of any perturbation' [1] during the contact between blood and the dialysis membranes or alternatively as an interacting body in which the definition of 'sum of specific interactions' [2] describes a more realistic view. This latter definition paves the way for an extension of the functional chart of hemodialyzer membranes that beside

\section{KARGER \\ Fax +4161306 1234 \\ E-Mail karger@karger.ch}

www.karger.com (c) 2012 S. Karger AG, Basel

0250-8095/12/0356-0559\$38.00/0

Accessible online at:

www.karger.com/ajn
Francesco Galli, $\mathrm{PhD}$

University of Perugia

Department of Internal Medicine

Via del Giochetto, IT-06126 Perugia (Italy)

Tel. +39075 585 7445, E-Mail f.galli@ unipg.it 


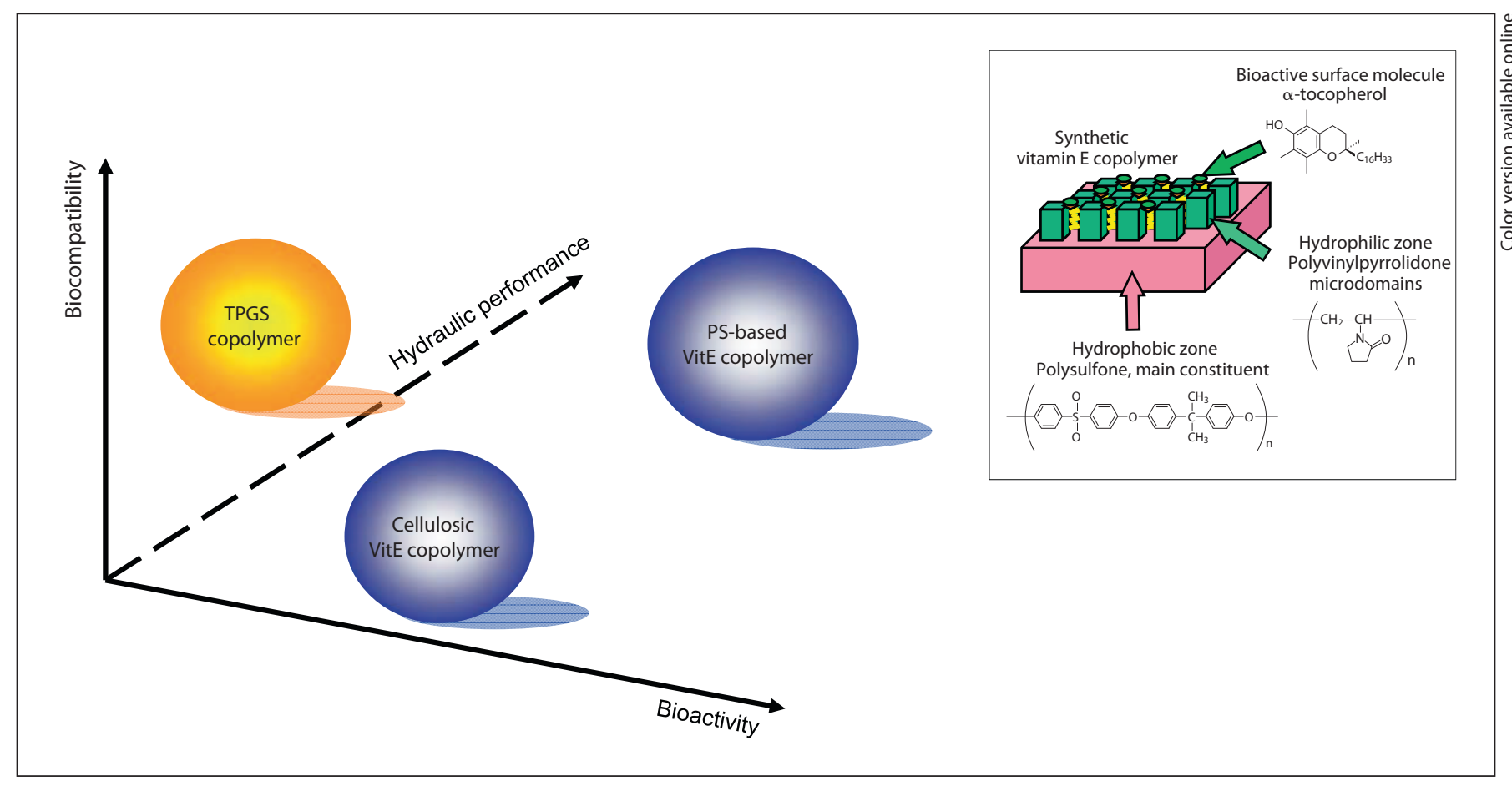

Fig. 1. 3D functional projection (right) and schematic structure (left) of the composite PS-polyvinylpyrrolidone copolymer of vitamin E-modified hemodialyzer membranes (VitabranE ${ }^{\mathrm{TM}}$ ).

filtration and biocompatibility may include bioactivity as a third feature (fig. 1, left panel).

The absorption of peptides and proteins such as $\beta_{2^{-}}$ microglobulin, cytokines and modified albumin is an example of how the interaction of HD membranes and blood constituents could be desirable rather than a problem. On the other hand, protein adsorption by the blood-membrane interaction has an impact on solute removal [3] and it cannot be ruled out that this could interfere with the structure/activity of normal or uremic solutes that include abnormal plasma proteins and small molecules such as the broad family of reactive carbonyls described below in this review paper. In that way, the dialyzer membrane may entrap on the blood surface solutes that reach high relative concentrations to become more available for the interaction with blood cell receptors and plasma proteins. These may result in untoward reactions and activation processes such as those described for components of the complement and coagulation pathways [4].

Further criteria to define the concept of biocompatibility ground on clinical classifications. Acute and chronic symptoms on the dialysis patient are the 'signature' of a bioincompatible material and the extent of these can predict the clinical outcome of a treatment. Biological and clinical signs of poor biocompatibility are consequent to immunoinflammatory and vascular reactions (see following section). Synthetic biomaterials with higher biocompatibility have constrained these effects to subclinical symptoms (essentially revealed by biochemical tests such as cytokines and acute-phase markers) thus contributing to alleviate long-term clinical consequences of HD therapy and uremia, such as protein catabolism and malnutrition [5] and a number of other chronic effects recently reviewed in [2]. Given the role that chronic inflammation and malnutrition play as risk factors for cardiovascular disease of chronic kidney disease (CKD), the use of biocompatible dialyzer membranes is expected to influence the cardiovascular outcome of patients on regular HD therapy, which is an assumption needing further support by randomized clinical trials [6].

However, even the highest standard in biocompatibility now available cannot avoid that dialyzer membranes and the entire extracorporeal circulation would provide immunoinflammatory reactions and chronic challenges to blood vessels. 
Fig. 2. Chemical structure of tocol and tocol-related compounds (4 tocopherols and 4 tocotrienols) appertaining to the family of vitamin $\mathrm{E}$.

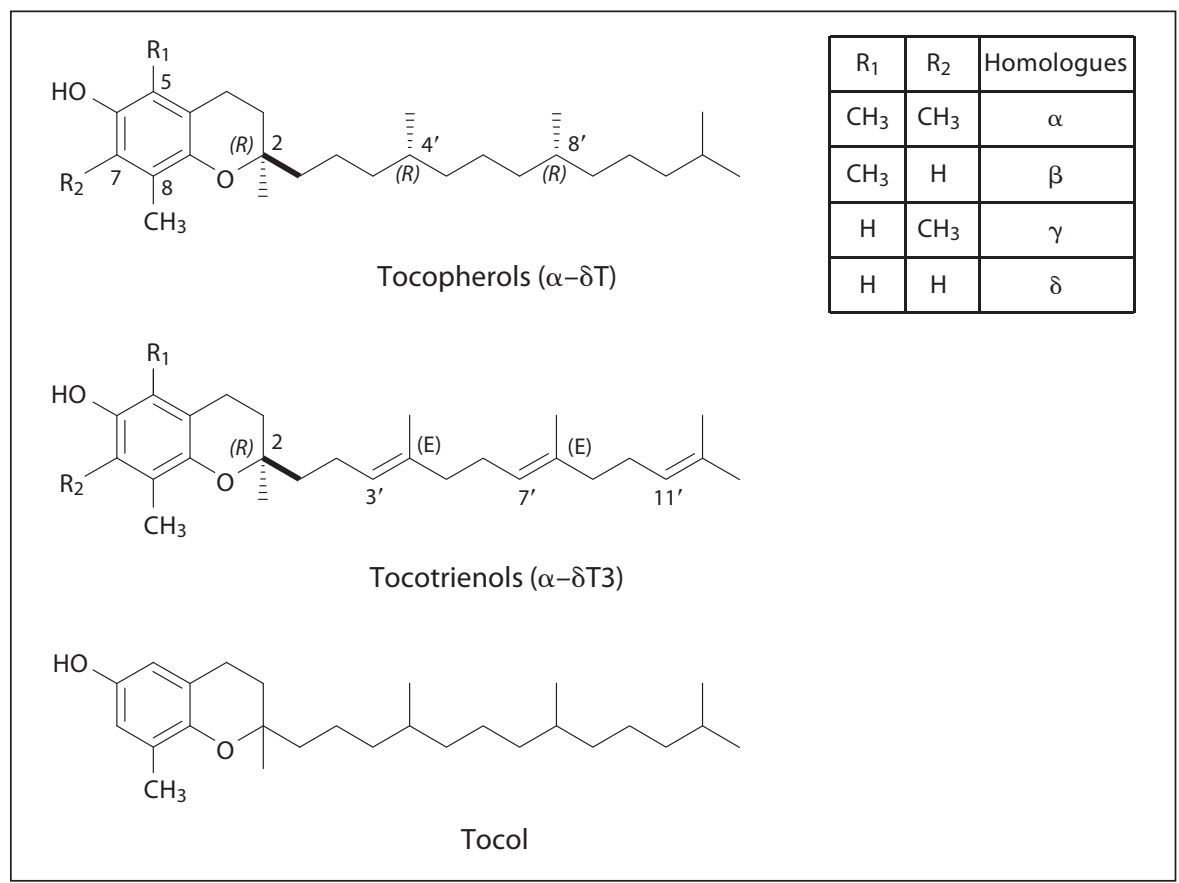

Poor biocompatibility of dialysis therapies results in proinflammatory, prooxidant stress and prothrombotic events. Accordingly, the repeated contact of patient's blood with the materials of the extracorporeal circulation causes oxidative stress biomarker formation, changes in the levels of inflammatory and anti-inflammatory cytokines (such as IFN- $\gamma$, TNF- $\alpha$, interleukin (IL)-1 $\beta$, IL-4, IL-6, IL-10, IL-12 and IL-18), acute-phase (C-reactive protein (CRP), SSA and fibrinogen) and negative-phase (serum albumin, prealbumin and transferrin) proteins, and leukocyte subsets, which reset to a dysfunctional immunoinflammatory phenotype [recently reviewed in 7]. Other consequences of bioincompatibility are the activation of complement and contact pathways $[4,8]$, and platelets [9]. Indices of red blood cell damage can represent other useful markers of biocompatibility $[7,10,11]$. The material of the hollow-fiber dialyzer membrane is the key player of blood cell activation together with bacterial contaminants in the dialysis fluids, which eventually come into contact with the patient's blood throughout the same dialyzer membrane. Vitamin E, as $\alpha$-tocopherol ( $\alpha$ TOC) (fig. 2), has been used to modify both cellulosic and polysulfone (PS) copolymers that are now available to produce hollow-fiber hemodialyzer membranes [recently reviewed in 12, 13] (fig. 1, right panel). This vitamin is used as a coating agent for HD biomaterials with the ultimate goal to mimic lipid structures of blood cell plas- malemma and lipoprotein particles, and to neutralize harmful reactive oxygen species (ROS). In fact, vitamin $\mathrm{E}$ is a well-known lipophilic antioxidant and a scavenger of hydroperoxyl radicals. Besides filtration and biocompatibility, this latter effect expands the functional definition of dialyzer membranes to a new dimension, i.e. that of 'antioxidant bioreactivity' described above and in the left panel of figure 1. Another example of this concept in HD biomaterials was that of heparin-grafted HD membranes [14]. Vitamin E can also be used as a template molecule to produce redox-silent (non-antioxidant) modifiers. Recently, D- $\alpha$-tocopheryl polyethylene glycol (PEG) 1000 succinate (TPGS), an esterified form of $\alpha$-TOC (fig. 3), was used to synthesize a PS-based copolymer, which appears to have improved in vitro biocompatibility and filtration performance [15].

These generations of vitamin E-derived copolymers have generated a new clinical perspective in HD therapy by developing more biocompatible biomembranes and even functional (redox-active) biomaterials. Early studies have provided preliminary clinical evidence in support of a clinical superiority of vitamin E-derived copolymers with respect to other synthetic biomaterials such as PS, which represent the gold standard in the production of cost-effective hollow-fiber hemodialyzers. Further and structured trials are awaited to achieve solid evidence. 


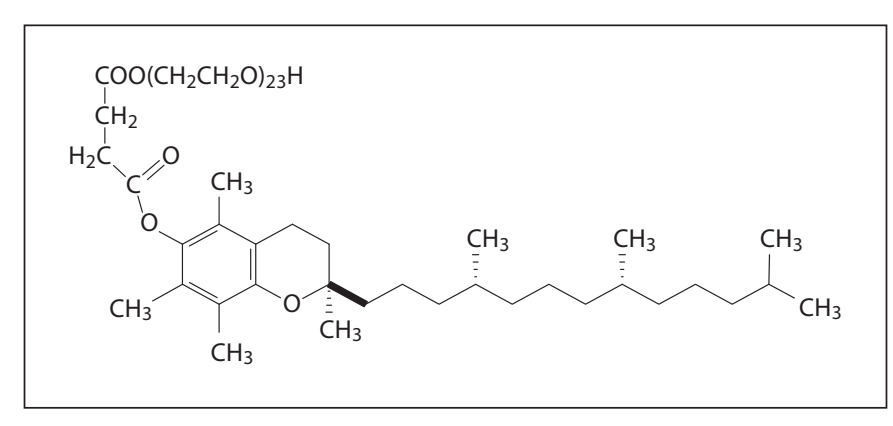

Fig. 3. Structure of TPGS, a synthetic derivative of vitamin E.

This review paper provides a critical examination of the literature so far produced on vitamin E-bonded dialyzer membranes.

\section{Bioincompatibility, Microinflammation and Oxidative Stress: Clinical and Biochemical Grounds}

Endothelial cells and the other cell components of arteries appear to have the greatest susceptibility to the challenging effects of chronic inflammation of CKD [reviewed in 16,17]. This may explain the increased cardiovascular morbidity and mortality of HD patients and the possible relationship with a poor biocompatibility of dialysis therapies [reviewed in 6].

Endothelial cells are highly prone to oxidative stress that can be prevented both in vitro and in vivo by antioxidants and mitochondrial protection agents [18-20]. ROS and receptor-dependent effects of cytokines and modified low-density lipoprotein (LDLs) are associated with an impaired energy function and apoptotic signaling of mitochondria, and cause ER stress through either Ca-dependent or -independent signaling in different types of cells $[21,22]$. At the same time, ROS and the binding of oxidized LDL (Ox-LDL) to scavenger receptors of endothelial cells activates the proinflammatory transcription factor as NF$\kappa \beta[23,24]$, inhibits the transcriptional activity of PPAR- $\gamma$ [25] and causes the accelerated senescence of endothelial progenitor cells [22]. These events altogether may sustain vascular microinflammation, arterial calcifications and atherosclerotic plaque formation by means of different underlying events such as (1) a defective capability of the arterial wall to prevent lipid deposition and foam cell formation, (2) a lowered capability to regulate the remodeling of the extracellular matrix and calcium deposition, (3) impaired mechanisms of prevention of leukocyte and platelet activation, and (4) poor repair of endothelial cell damage.
If these factors promote the formation of inflammatory lesions of the main vessels, other tissues such as the skeletal muscle and liver undergo a series of changes mediated by cytokines such as TNF- $\alpha$ and IL- 6 . These also sustain a negative protein metabolism in these tissues, which is a major causal event in CKD malnutrition [26] and may also contribute to insulin resistance [27]. Lipoperoxidation by-products such as oxysterols and 4-hydroxynonenal conspire with direct effects of oxidation to impair the endocrine and paracrine function of the adipose and skeletal muscle. These products may affect the levels and lipotoxicity of free fatty acids and adipokines and ultimately may cause insulin resistance. A defective insulin signaling impairs the endothelial metabolism of nitric oxide and the activity of plasminogen activator inhibitor-1, which together with platelet activation, increase thrombogenicity in patients treated with bioincompatible materials. RBC rheology may change upon bioincompatible treatments further increasing cardiovascular risk.

Oxidative and non-oxidative events triggered by the extracorporeal treatments are believed to influence the entire uremic comorbidity. Beside cardiovascular disease, chronic inflammation contributes to uremic anemia by lowering the responsiveness to erythropoietinstimulating agent (ESA) and erythrocyte life span [28, 29]. Leukocyte activation and oxidative stress are also reported to cause erythrocyte damage that is sustained by shear stress and other mechanical injuries.

The repeated activation of leukocytes by the contact with bioincompatible membranes is a cause of leukopenia $[30,31]$ and is a leading cause of the defective cell-mediated immune response of HD patients that is associated with the conversion of lymphocytes from Th1 to Th2 phenotype and with an overproduction of inflammatory cytokines [7].

The release of pyrogens and bioactive mediators such as the vasoactive components histamine and bradykinin contribute to fever and intradialytic hypotension which are other common intradialytic events associated with the treatment with bioincompatible materials [reviewed in 32,33$]$.

Chronic challenges to the immune system by bioincompatible dialysis therapies can worsen the prognosis of CKD patients leading to a condition of systemic microinflammation. A self-feeding mechanism with activation loops centered on leukocytes and vascular cells has been proposed to explain the pathogenesis of CKD microinflammation [16]. These loops are fed through the same products of inflammation, i.e. cytokines and ROS [34], which per- 
petuate the stimulation of inflammatory and vascular cells in combination with uremic factors and with the abnormal generation of by-products of ROS and carbonyls [35].

The burden of oxidative stress-derived by-products in CKD is higher than in other conditions of chronic inflammation, reaching the highest levels in end-stage renal failure patients. In CKD, unsaturated lipids show higher than normal oxidizability when exposed to peroxidizing agents [36-38] and plasma proteins show signs of damage by endogenous pro-oxidants, nitrative/nitrosative agents, and carbonylation by the reaction with lipid oxidation and glycation by-products [reviewed in 17, 34, 39-41]. Small solutes such as reactive carbonyls and larger solutes such as post-translational modifications of proteins, form a plethora of uremic solutes and immunoinflammatory mediators that contribute to sustain microinflammatory loops with different and only partially understood mechanisms. These solutes are the expression of a component of the uremic toxicity that is largely unaffected by the current dialysis strategies [42]. In fact, diffusive, convective or mixed techniques provide poor removal of middle to large solutes generated by the action of ROS and reactive carbonyls [reviewed in 34, 4346].

In the end, these proinflammatory solutes sustain cell and tissue reactions through receptor-dependent and -independent mechanisms of scavenging that are responsible of the endogenous metabolism of these by-products. Modified apolipoproteins and other plasma proteins bearing post-translational modifications, such as glycated albumin [47], are scavenged by the activity of specific surface receptors, such as RAGE and the homologue CD36 that is mainly involved in Ox-LDL scavenging. These receptors are expressed in mononuclear leukocytes, neutrophils and endothelial cells, e.g. the same cells that upon stimulation produce ROS, inflammatory cytokines, chemokines, adhesion molecules and acutephase mediators, and provide the ultimate explanation to the self-feeding loop that sustains the microinflammatory syndrome of HD patients [34].

\section{Strategies to Face Bioincompatibility: Is Antioxidant Therapy Included?}

To reduce the risk of oxidative stress and other adverse events caused by the poor biocompatibility of HD treatment, highly purified dialysis fluids and generations of synthetic dialyzer membranes have been developed.
Bioincompatibility of prototypical dialyzer membranes made of regenerated cellulose has been associated with the contact of blood components with the hydroxyl groups of the $\beta$-D-glucose structure. These groups trigger intradialytic activation of the complement system and cause leukopenia [31]. Based on this burden of knowledge, two major approaches have been adopted to improve the biocompatibility of regenerated cellulose dialyzer membranes. One was chemical modification of the hydroxyl groups, e.g. modification by acetylation to obtain triacetate cellulose or with PEG chains to obtain PEG-grafted cellulose. The other, and more successful, approach was based on the development of synthetic polymer membranes such as PS or polyether sulfone membranes, which have no bioincompatible groups in their chemical structures $[48,49]$. Nowadays, high-flux synthetic membranes are more frequently used and represent a cost-effective solution with proven clinical superiority over cellulosic membranes.

At the same time, symptomatic approaches have been proposed on the side of oxidative stress. Antioxidants such as vitamins $\mathrm{C}$ and $\mathrm{E}$, and glutathione, have been used as oral supplements in an attempt to correct the progressive decrease of blood antioxidants and to alleviate the abnormal generation of ROS of HD patients [reviewed in 35] (fig. 4). In fact, CKD is a condition of antioxidant deficiency and particularly of water-soluble antioxidants (such as vitamin C) that undergo chronic leakage by the HD treatments. CKD is also a condition of higher antioxidant demand by the proinflammatory and oxidative stress environment described in the previous sections. This higher demand can be deduced by the higher susceptibility to lipid oxidation of uremic plasma and the decreased content of blood cell antioxidants [36, 50]. Few studies, however, provided solid evidence of a clinical advantage of the oral administration of antioxidants. The most convincing evidence came from the randomized clinical trial on vitamin E supplementation in HD patients known as the 'SPACE study' [51] that demonstrated a significant reduction (54\%) of the primary endpoint (a composite variable including myocardial infarction, ischemic stroke, peripheral vascular disease, unstable angina) and $70 \%$ reduction in myocardial infarction. To confirm CKD as an elective condition for antioxidant therapy, other successful small-scale trials have been reported in CKD [reviewed in 11, 74].

One of the most original approaches of the antioxidant therapy in HD involved the use of vitamin E (fig. 2), a well-known lipophilic antioxidant, as a modifier of the surface of the dialysis membrane that is described in the 
Fig. 4. Average change of some indices of oxidative stress in plasma along the clinical progression of CKD. Data are as percent of mean healthy control values. All the groups have $n=10$, except of group 1 $(\mathrm{n}=11)$ and group $2(\mathrm{n}=8)$. TAC $=$ Total antioxidant capacity; $\mathrm{UA}=$ uric acid; $\mathrm{AA}=$ ascorbic acid; $\mathrm{CrCl}=$ creatinine clearance [from F. Galli, unpubl. data].

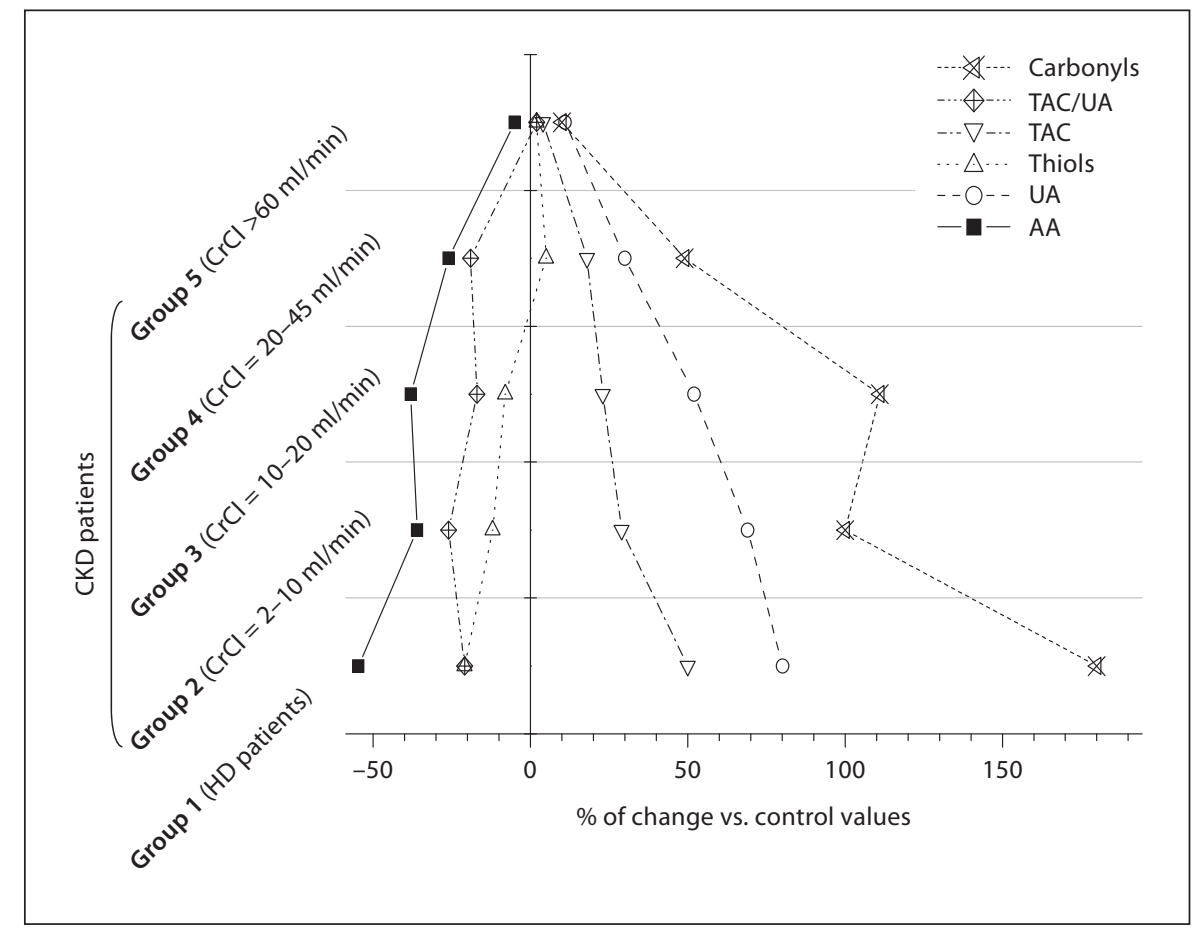

following sections. The bonded vitamin E on the surface of the dialyzer membrane is expected to reduce the production of ROS at the site where blood cells come into contact with the dialyzer membrane, thereby providing antioxidant protection in a timely and targeted manner. Because of this, two generations of vitamin E-coated dialyzers, namely cellulosic- and synthetic polymer-based membrane dialyzers, were successfully developed and launched on the market in the last two decades.

\section{General Concepts on Biology and Chemistry of Vitamin E}

Vitamin E was discovered in 1922 when Evans and Bishop [52] described a 'substance X' essential for rat fertility. A family of 8 structurally related compounds (4 tocopherols and 4 tocotrienols) is included under the name of vitamin E. Their basic structure is that of tocochromanols (fig. 2, lower structure) that in the prototypal form of $\alpha$-TOC brings a 6-hydroxychroman moiety and a phytyl side chain attached at carbon 2 (fig. 2, upper structure). Tocopherols contain saturated side chains and three asymmetric centers (positions 2, $4^{\prime}$ and $8^{\prime}$ ) while tocotrienols (fig. 2, middle structure) have 3 double bonds in the positions $3^{\prime}, 7^{\prime}$ and $11^{\prime}$ and one asymmetric center (position 2). The methylation pattern of the chroman ring gives origin to 4 homologues in both the tocopherol or tocotrienol families identified with the Greek letters $\alpha, \beta$, $\gamma$, and $\delta$. Natural tocopherols (and tocotrienols) show right-handed configuration $(\mathrm{R})$ in all the asymmetric (or chiral) centers, and thus are identified as RRR optical isomers, while synthetic forms are racemic mixtures (as the all-rac- $\alpha$-TOC included in many vitamin $\mathrm{E}$ supplements) containing all the different combinations of the $\mathrm{R}$ and left-handed (S) optical configurations.

Humans and animals are unable to synthesize vitamin $\mathrm{E}$ and must obtain it from plant sources (vegetable oils, dressings, shortenings and margarines) that often contain at the same time various members of the vitamin $\mathrm{E}$ family [53]. Vitamin E, like cholesterol, is found in almost all organs. Approximately $3 \mathrm{~g}$ of vitamin $\mathrm{E}$ is stored in the body of a healthy adult. The main reserves are in subcutaneous fat, muscles, and the liver. The concentration of vitamin E per gram of tissue is high in adipose tissue (150 $\mu \mathrm{g} / \mathrm{g})$, in the adrenal glands $(132 \mu \mathrm{g} / \mathrm{g})$, in the pituitary or hypophysis $(40 \mu \mathrm{g} / \mathrm{g})$ and in the testes $(40 \mu \mathrm{g} / \mathrm{g})$.

Since its discovery, $\alpha$-TOC has been considered the most important molecule of vitamin E family [54]. It is the most abundant form of vitamin $\mathrm{E}$ in blood and several tissues, followed by $\gamma-\mathrm{TOH}$, while the other vitamins are present only in traces. At the same time, $\alpha$-TOC 
proves more active than any of the other 'non- $\alpha$ ' homologues when assessed with several biological tests. Indeed, the biological activity calculated by the classical test of fetal reabsorption in the rat gives values ranging from $100 \%$ for $\alpha$-TOC to $30 \%$ for $\beta$-TOC and $1.4 \%$ for $\delta$-TOC [55]. Nowadays, vitamin E supplements almost exclusively contain $\alpha-\mathrm{TOH}$. Emerging evidence is demonstrating the different contribution of minor forms, such as $\gamma-\mathrm{TOH}$ and tocotrienols, in the biological responses elicited by the vitamin E family of compounds [reviewed in 54, 5658]. These include anticancer, anti-inflammatory and immunomodulatory effects, lipid-lowering and neuroprotection functions.

One of the key biological roles of vitamin $\mathrm{E}$ is that of a physiological liposoluble antioxidant trapping peroxyl radicals and other reactive species generated during cell metabolism and oxidative stress [59,60]. Antioxidant roles of vitamin $\mathrm{E}$ are influenced by the composition of the reaction medium which may provide different chemical interactions for instance with targets and particularly with co-antioxidants that include other lipophilic and hydrophilic antioxidants with biological relevance in cells and biological fluids such as ascorbic acid and glutathione $[61,62]$. Therefore, the study of the chemistry of vitamin E oxidation is of considerable interest in connection with its mode of action and physiological relevance. The one-electron oxidation of $\alpha$-TOH provides the production of a tocopheroxyl radical that can further react with lipid peroxides, leading to the formation of 8-substituted tocopherones (hydroxy- or alkyldioxytocopherones), tocopheryl quinone (TQ), epoxyquinones (TQE1 and TQE2) and eventually tocopherol hydroquinone [63]. The analysis of these compounds has been used to give a measure of the oxidation of membrane lipids and also to test antioxidant activity of vitamin E-modified hollowfiber dialyzer [64].

\section{Vitamin E-Bonded Dialyzers}

\section{Development of Cellulose-Based Vitamin E-Bonded Dialyzer Membranes}

In 1990, cellulose-based vitamin E-bonded dialyzer membranes were developed and introduced on the market by Terumo Corporation under the commercial name Excebrane $^{\mathrm{TM}}$. The base membrane was regenerated cellulose hollow fiber, and the surface of the hollow fiber was covalently modified by hydrophilic polymers as well as oleic alcohol; then, vitamin E ( $\alpha$-TOC) was bonded to oleic alcohol via hydrophilic interaction. Sasaki et al. [65] reported the outline of the production process as well as the results of in vitro and in vivo studies on Excebrane, which showed much better biocompatibility than the original regenerated cellulose membrane.

\section{Clinical Outcome of Cellulose-Based Vitamin \\ E-Bonded Membranes}

Early trials using Excebrane indicated positive clinical effects [reviewed in 13, 32, 66]. Reduction of oxidative stress and inflammation was described as the possible therapeutic effect. Cardiovascular endpoints were investigated in several small clinical trials and a meta-analysis of 14 peer-reviewed articles by Sosa et al. [66] concluded that Excebrane treatment was associated with a significant decrease of lipid peroxidation biomarkers in plasma, thereby suggesting the potential benefit of these membranes in clinical usage. Actually, these biomarkers are associated by a cause-effect relationship with LDL damage and endothelial dysfunction in CKD. Preliminary evidence of a better management of uremic anemia by these dialyzer membranes was obtained in the pioneering studies of Usberti et al. [67] in 2002 and by other authors thereafter [68, 69]. These authors found improved erythrocyte life span and rheology in patients on Excebrane treatment. A larger multicenter study (172 patients) by Cruz et al. [70] confirmed the better impact of Excebrane dialyzers on anemia parameters when compared with other high-flux biocompatible dialyzer membranes, including cellulose acetate, PS, and polymethylmethacrylate [also reviewed in 71].

Other reports demonstrated the positive effects related to (a) decreased oxidative stress [72-81]; (b) suppression of leukocyte activation [82-88]; (c) reduction of anticoagulant dosage [89]; (d) improved biocompatibility [90], and (e) decreased levels of advanced glycation end products [91]. Moreover, a recent study by Kirmizis et al. [92] showed lowered levels of the inflammatory markers CRP, IL-6, and soluble intercellular adhesion molecule-1 in 35 patients when treated with Excebrane with respect to baseline treatments carried out with conventional lowor medium-flux dialyzers, while no change was observed in a matched control group of 25 patients who underwent treatment with these later unmodified dialyzers for the whole observation time.

In 2010, a small $(n=9)$ observational A-B study on vitamin E-coated cellulose acetate membranes compared with PF membranes [93] reported an improved and longterm control of inflammatory (high-sensitivity CRP, total antioxidant capacity, and IL-6) and oxidative stress (d-ROM test and superoxide dismutase) biomarkers. 
Few studies have compared oral supplementation of vitamin E and the treatment with Excebrane dialyzers. A pioneering study by Akiyama et al. [81] showed the same effect of these treatments on the expression of the antioxidant enzyme $\mathrm{Cu}-\mathrm{Zn}$ superoxide dismutase in circulating mononuclear leukocytes of HD patients, which may represent a new in vivo oxidative stress biomarker associated with lipid oxidation of leukocyte plasmalemma [80]. Interestingly, this biomarker does not appear to be affected by the oral supplementation with the hydrosoluble antioxidant vitamin C [94]. This early evidence paves the way to a specific role of vitamin $\mathrm{E}$ therapy in the protection of circulating leukocytes, which deserves further clinical investigation in $\mathrm{CKD}$ patients on regular $\mathrm{HD}$ therapy.

\section{PS-Based Vitamin E-Bonded Membranes}

Synthetic membrane dialyzers have been developed by many manufacturers in recent decades to provide higher depurative and biocompatibility standards. PS has shown better performance among these synthetic biomaterials; as a result, it has gained popularity in clinical practice. In order to synergize biocompatibility of synthetic membranes and antioxidant activity of vitamin E, PS-based vitamin E-coated membranes have been developed.

First introduced on the Japanese market in 2000, these PS-based dialyzers were initially developed by Terumo Corporation, and are now manufactured using a new technique by Asahi Kasei Kuraray Medical Co. Ltd under the commercial name VitabranE ${ }^{\mathrm{TM}}$. The actual backbone of this membrane is a composite PS-polyvinylpyrrolidone copolymer (fig. 1) that has been developed to produce optimum flow dynamics and clearance rates. Preliminary in vitro analyses on these membranes were reported by Sasaki [13] and Floridi et al. [64]. This new type of vitamin $\mathrm{E}$ membrane has generated great interest and expectations $[71,95]$ that however has to be considered within a careful analysis of biomaterial characteristics, biological mechanisms and the outcome of clinical trials [96]. Available knowledge of these aspects is reported below.

First Evidence of Vitamin E-Bonded Membranes as

'Antioxidant Biomaterial'

The antioxidant capacity of VitabranE was recently confirmed and quantified by Floridi et al. [64] by means of in vitro recirculation tests carried out on mini-module dialyzers. These tests, which used an unbiased procedure, showed that at least one-third of the vitamin E present on the membrane participates to one electron transfer reac- tion with transition metals. This reaction, together with scavenging of peroxyl radicals, characterizes the antioxidant mechanism and is of putative relevance in the biological function of vitamin E.

\section{Clinical Outcome of Vitamin E-Bonded PS \\ Membranes}

Anemia

Based on early evidence obtained in Excebrane studies described above, VitabranE was proposed to help controlling anemia of HD patients. This aspect was investigated in clinical trials that compared VitabranE with PS membranes. A pilot study by Andrulli et al. [97] carried out with a controlled randomized two-arm design, showed that the 8-month treatment with VitabranE decreased the ESA resistance index (ERI), as calculated by the ratio between ESA dosage (IU/kg/week) and hemoglobin levels $(\mathrm{g} / \mathrm{dl})$. This effect became statistically significant in the comparison with the PS group, when the baseline parathyroid hormone and serum vitamin E levels were included as covariates in the secondary analysis. The authors concluded that VitabranE membranes have a possible beneficial effect on ERI of HD patients [97]. This conclusion was confirmed in another and more recent multicenter study by Panichi et al. [98]. In this report, $62 \mathrm{HD}$ patients from 13 dialysis units were randomized in a crossover design of treatments with VitabranE and control PS dialyzers. The patients were studied for two steps of 6 months in each treatment. In this study, hemoglobin levels significantly increased after 6 months of VitabranE treatment, while these remained unchanged in the control PS dialyzer group. Moreover, at the same ESA dose, the ERI was significantly lower in the VitabranE treatment period.

Further support for the findings of these randomized trials came from a pilot crossover study by Mandolfo et al. [99] conducted on patients using central venous catheters for blood access. The 16 patients enrolled and divided into two treatment groups (VitabranE dialyzer vs. synthetic membrane dialyzers) were followed for two time periods of 6 months each. The results showed that the ERI decreased significantly in the treatment with VitabranE, while no change was observed in the control treatment. Hemoglobin levels were not affected and thus the authors concluded that VitabranE membranes may help to control antianemic therapy decreasing the dosage of ESA.

Large randomized controlled trials on anemia management are being independently performed in Japan, the $\mathrm{UK}$, and Italy. 
Reduction of Inflammatory Markers

In the report by Panichi et al. [98], the authors observed that in addition to the reduction of ERI, CRP and IL-6 levels were significantly decreased on VitabranE treatment, but not on PS treatment. The study by Mandolfo et al. [99] on catheterized patients with a higher inflammatory burden also reported better control of CRP levels during the VitabranE treatment. In these studies, a lower inflammatory response was associated with improved ERI, which is consistent with the role of chronic inflammation and oxidative stress as pathogenic factors of uremic anemia [33].

Calò et al. [100] conducted a 1-year study on $25 \mathrm{HD}$ patients to evaluate the effects of VitabranE on biochemical markers of inflammation. The authors used a molecular approach (immunoblot analysis) to assess at the beginning of the study and after 6 and 12 months of treatment with VitabranE the expression of p22 (phox), heme oxygenase-1, plasminogen activator inhibitor and phosphorylated extracellular signal-regulated kinase-1/2 in circulating mononuclear leukocytes. The treatment with VitabranE significantly decreased the expression of these protein markers that are relevant to inflammation.

Furthermore, preliminary investigations of the levels of advanced protein oxidation products and fluorescent glycation end products were performed in the randomized controlled pilot trial reported by Andrulli et al. [97]. These were investigated as oxidative and carbonyl stress indices associated with the correction of ERI. Although IL- 6 and CRP levels were not affected in this study, the two indices of protein damage showed a better control by the treatment with VitabranE than PS dialyzer [Galli et al., in preparation].

\section{Reduction of Oxidative Stress Markers}

Vitamin E-bonded dialyzer membranes were originally developed with the aim of reducing oxidative stress. As shown above, lowered lipid peroxidation was observed in clinical trials on vitamin E-bonded cellulose-based membrane. With regard to VitabranE, two studies have reported on LDL oxidation as a laboratory endpoint. In 15 patients who received 6 months of treatment with VitabranE, Morimoto et al. [101] showed a significant reduction in the levels of asymmetric dimethylarginine, malondialdehyde LDL, and Ox-LDL. The levels returned to baseline when the membrane was changed to a PS dialyzer membrane. In a matched control group, patients treated with PS dialyzers showed no change in asymmetric dimethylarginine, Ox-LDL, and malondialdehyde LDL levels during the entire treatment period of 18 months. The other report from Calò et al. [100] showed that VitabranE improved the levels of inflammation markers also reducing plasma levels of Ox-LDL, which was evaluated by enzyme-linked immunosorbent assay.

Another small randomized study [102], however, failed to observe a different extent of LDL oxidizability in patients treated for 3 months with vitamin E-coated membranes that were compared with perspective crossover design to reference PS membrane. At the same time, however, the authors reported for this multicenter study that LDL oxidizability showed marked intercenter (or regional) variability, which may suggest the introduction of local biases in the selection of patients and treatments. As a consequence, the results of the comparison between the different membranes assessed in this study should be considered with caution.

\section{Intradialytic Hypotension}

Intradialytic hypotension (IDH) is a common clinical trait in HD. Matsumura et al. [103] conducted a pilot study to assess the effectiveness of VitabranE in improving IDH. Eight IDH patients were switched from their conventional dialyzers to a VitabranE dialyzer, and intradialytic blood pressure (BP) was monitored regularly for 10 months. The results showed that hypotension, as monitored during the session by measuring systolic BP, diastolic BP, and pulse pressure, improved after changing the dialyzer membrane. Moreover, after 8-10 months, systolic BP recorded before dialysis was significantly lower than at baseline, which suggests a stable improvement in the vascular compliance to intra- and interdialysis control of BP.

\section{Anticoagulation Management}

In a recent report, Aoun et al. [104] described possible clinical advantages of VitabranE in anticoagulant management. In an observational trial, these authors evaluated the minimum requirement of low-molecular-weight heparin in pediatric dialysis patients. Seven children and adolescent patients were started on VitabranE dialyzer and their low-molecular-weight heparin dose was decreased every week without any other changes in the clinical management; the findings of this study consistently indicated a lower requirement of anticoagulants, which may contribute to reduce bleeding problems and simplifying hemostasis in post-dialysis. 
Other Vitamin E Copolymers for Possible Application in HD Therapy

Synthetic analogues of vitamin E can be used as bonding agents to develop a variety of vitamin E-modified biocopolymers. In a recent study published in this journal by Dahe et al. [15], a new PS-based hollow-fiber membrane incorporating from 5 to 20\% (w/w) TPGS was described (fig. 3). The authors claimed this biomaterial as 'antioxidative composite' PS (see the Abstract) and conclude that 'we have successfully incorporated biologically active vitamin E TPGS.... Biocompatibility and separation performance were assessed with different methods and the reported results are summarized by the authors in terms of 'enhanced biocompatibility ... than commercial hemodialysis membranes'.

It is worth noting that TPGS has completely different properties when compared with authentic RRR $\alpha$-TOC. First of all, TPGS is a redox-silent molecule used to prepare copolymers and drug excipients. This means that TPGS is not an 'antioxidative' molecule. Chroman ring succinylation can occur only in position 6 by esterification of the hydroxyl moiety [to visualize structural features of synthetic vitamin E derivatives, see e.g. 57, 105], which prejudices the radical scavenging and electron-donating properties of $\alpha$-TOC [57]. Such an esterification reaction together with the presence of the PEG 1000 moiety deeply modify the canonical chemical and physical characteristics of vitamin E. Partition coefficient in lipidwater matrices, steric properties and the affinity for lipidbinding proteins (such as $\alpha$-tocopherol transfer protein or other low affinity-binding proteins) as well as the interaction with phospholipids and cholesterol in membranes and lipoproteins, are all deeply modified with respect to vitamin $\mathrm{E}$. This means that TPGS may have better biocompatibility according to the reported findings in this study, but cannot be defined as an 'antioxidative' biomaterial. Also the definition 'biologically active vitamin E TPGS' is speculative since the most likely biological consequence of incorporating TPGS into PS is that of producing and inert (less bioactive) biomaterial. The possibility that TPGS would be released from the composite PS may offer a chance for its de-esterification by cellular esterases to release free (and thus bioactive) vitamin E, but this chance was not investigated in this study in which TPGS appears to be stabilized in the fiber structure. Based on a legal view, the release of TPGS and its endogenous activation by esterases may lead to define these membranes as a prodrug-modified medical device, which might be categorized to 'medicine' and not 'medical device'.
Bioactivity of TPGS-PS was also investigated by means of in vitro biocompatibility tests that included the analysis of ROS generated in cells maintained in culture with the modified fibers, which improved in this new biomaterial. A lower generation of these species could be claimed as an 'antioxidative' effect even if this is an indirect event that is secondary to a lower cell activation (NADPH oxidase or mitochondria-dependent ROS could be involved). Once again, the investigation of TPGS bioavailability (with fiber release and cell uptake tests) as well as a control experiment with free TPGS would help to verify the results in these tests and the specificity of TPGS bioactivity, if any.

This response on ROS generation is expected, but was not proven in this study, to mirror lower leukocyte activation in the extracorporeal circulation, ultimately representing an indirect (or secondary) antioxidant effect due to TPGS-PS biocompatibility. However, it is difficult to guess how such a cell experiment together with other similar in vitro biocompatibility tests reported in this study, may reproduce the expected in vivo interaction (or contact) of blood cell plasmalemma with the inner surface of the fibers that were shown with different techniques to incorporate TPGS. As a likely event, cells came into contact with the external surface of the fibers which may have a different composition and morphology with respect to the inner surface.

Notwithstanding, this indirect 'antioxidative' effect and the other results of biocompatibility tests are promising and deserve further investigation to confirm whether the 'enhanced biocompatibility ... than commercial hemodialysis membranes' can be confirmed in a suitable extracorporeal circulation model system with appropriate controls and simulation of the in vivo conditions.

In a recent study by our group [64], the actual antioxidant power of vitamin $\mathrm{E}$ was assessed in the vitamin E-modified hollow-fiber PS membranes produced with another technology and using $\alpha$-TOC as modifier. This aspect was not touched in the study by Dahe et al. [15], and this appears appropriate once it is recognized that TPGS-PS is a redox-silent biomaterial, which further confirms the fallacy of claiming this as antioxidative composite' PS.

The coating with vitamin $\mathrm{E}$ in the hemodialyzer membranes currently in use in HD centers has been proposed to prevent LDL oxidation [reviewed in 32,66]] and to alleviate the resistance to erythropoiesis-stimulating agents $[97,98]$, leading to speculate antioxidant effects for near to two decades of clinical trials. Only after such an accurate in vitro evaluation carried out by means of a recircu- 
lation-based assay were these membranes undoubtedly demonstrated to be 'antioxidative'. On the basis of these considerations, we would like to highlight that this claim does not apply for TPGS-PS membranes described in the study of Dahe et al. [15].

Further and more accurate studies on vitamin E-derived bonding agents are awaited since these may lead to the development of future generations of copolymers with possible application in the manufacturing of biocompatible and bioactive dialyzer membranes.

\section{Conclusion}

Vitamin E bonding on the cellulosic membrane, Excebrane, has suggested to reduce oxidative stress, as assessed by lipid peroxidation markers, and to improve laboratory indices of inflammation and uremic anemia. The newly developed synthetic PS-based dialysis membrane, VitabranE, allowed more relevant clinical comparisons being homologous with most widely used synthetic membrane dialyzers. Randomized studies carried out in the last few years have reported positive effects on ERI and anemia management. This represents the most convincing and clinically relevant evidence so far obtained with VitabranE. Early findings also suggest a better control of inflammatory and oxidative stress parameters. Anticoagulation and IDH are other aspects of possible relevance in the clinical application of VitabranE. However, only a limited number of small-scale pilot studies have reported on this new vitamin E-bonded membrane, and only a few of them have been conducted with a randomized controlled design. These limits do not allow drawing conclusive clinical inferences on these membranes; nevertheless, available results are suggestive of a superior performance with respect to other synthetic membranes. Further and more comprehensive trials are awaited to verify these results.

\section{Disclosure Statement}

Dr. Aritomi is an employee of a company that manufactures and sells HD membranes of the type described in this article. The results presented in this paper have not been published previously in whole or part.

\section{References}

1 Cheung AK: Biocompatibility of hemodialysis membranes. J Am Soc Nephrol 1990;1: 150-161.

2 Takemoto Y, Naganuma T, Yoshimura R: Biocompatibility of the dialysis membrane. Contrib Nephrol. Basel, Karger, 2011, vol 168, pp 39-145.

- 3 Huang Z, Gao D, Letteri JJ, Clark WR: Blood-membrane interactions during dialysis. Semin Dial 2009;22:623-628.

$\checkmark 4$ Mares J, Richtrova P, Hricinova A, Tuma Z, Moravec J, Lysak D, Matejovic M: Proteomic profiling of blood-dialyzer interactome reveals involvement of lectin complement pathway in hemodialysis-induced inflammatory response. Proteomics Clin Appl 2011;4:829-838.

5 Parker TF 3rd, Wingard RL, Husni L, Ikizler TA, Parker RA, Hakim RM: Effect of the membrane biocompatibility on nutritional parameters in chronic hemodialysis patients. Kidney Int 1996;49:551-556.

6 Rabindranat KS, Muirhead N: Modalities of extracorporeal therapy: hemodialysis, homofiltration, and hemodiafiltration; in Molony DA, Craig JC (eds): Evidence-Based Nephrology. Oxford, Wiley-Blackwell, 2009, pp 410-422.
7 Libetta C, Sepe V, Esposito P, Galli F, Dal Canton A: Oxidative stress and inflammation: implications in uremia and hemodialysis. Clin Biochem 2011;44:1189-1198.

-8 Amore A, Coppo R: Immunological basis of inflammation in dialysis. Nephrol Dial Transplant 2002;17(suppl 8):16-24.

-9 Brunini TM, Mendes-Ribeiro AC, Ellory JC, Mann GE: Platelet nitric oxide synthesis in uremia and malnutrition: a role for L-arginine supplementation in vascular protection? Cardiovasc Res 2007;73:359-367.

10 Galli F, Rovidati S, Benedetti S, Buoncristiani U, Covarelli C, Floridi A, Canestrari F: Overexpression of erythrocyte glutathione S-transferase in uremia and dialysis. Clin Chem 1999;45:1781-1788.

11 Stoya G, Klemm A, Baumann E, Vogelsang $\mathrm{H}$, Ott U, Linss W, Stein G: Determination of autofluorescence of red blood cells in uremic patients as a marker of oxidative damage. Clin Nephrol 2002;58:198-204.

12 Aritomi M, Galli F: Review of the effectiveness of cellulose - and polysulfone-based vitamin E-bonded dialysis membranes; in Penido MG (ed): Hemodialysis. InTech, Open Access Publisher, 2011: Technical Problems in Patients on Hemodialysis, Book 4.
13 Sasaki M: Development of vitamin E-modified polysulfone membrane dialyzers. J Artif Organs 2006;9:50-60.

14 Morena M, Jaussent I, Chalabi L, Bargnoux AS, Dupuy AM, Badiou S, Rakic C, Thomas M, Canaud B, Cristol JP: Biocompatibility of heparin-grafted hemodialysis membranes: impact on monocyte chemoattractant protein-1 circulating level and oxidative status. Hemodial Int 2011;14:403-410.

$\checkmark 15$ Dahe GJ, Teotia RS, Kadam SS, Bellare JR: The biocompatibility and separation performance of antioxidative polysulfone/vitamin E TPGS composite hollow fiber membranes. Biomaterials 2011;32:352-365.

16 Mizobuchi M, Towler D, Slatopolsky E: Vascular calcification: the killer of patients with chronic kidney disease. J Am Soc Nephrol 2009;20:1453-1464.

17 Himmelfarb J: Uremic toxicity, oxidative stress, and hemodialysis as renal replacement therapy. Semin Dial 2009;22:636-643.

18 Dhanasekaran A, Kotamraju S, Kalivendi SV, Matsunaga T, Shang T, Keszler A, Joseph J, Kalyanaraman B: Supplementation of endothelial cells with mitochondria-targeted antioxidants inhibit peroxide-induced mitochondrial iron uptake, oxidative damage, and apoptosis. J Biol Chem 2004;279:37575-37587. 
19 Messner B, Zeller I, Ploner C, Frotschnig S, Ringer T, Steinacher-Nigisch A, Ritsch A, Laufer G, Huck C, Bernhard D: Ursolic acid causes DNA damage, p53-mediated, mitochondria- and caspase-dependent human endothelial cell apoptosis, and accelerates atherosclerotic plaque formation in vivo. Atherosclerosis 2011;219:402-408.

-20 Rocha M, Apostolova N, Hernandez-Mijares A, Herance R, Victor VM: Oxidative stress and endothelial dysfunction in cardiovascular disease: mitochondria-targeted therapeutics. Curr Med Chem 2010;17:3827-3841.

-21 Gurgul-Convey E, Lenzen S: Protection against cytokine toxicity through endoplasmic reticulum and mitochondrial stress prevention by prostacyclin synthase overexpression in insulin-producing cells. J Biol Chem 2011;285:11121-11128.

-22 Carracedo J, Merino A, Briceno C, Soriano S, Buendia P, Calleros L, Rodriguez M, Martin-Malo A, Aljama P, Ramirez R: Carbamylated low-density lipoprotein induces oxidative stress and accelerated senescence in human endothelial progenitor cells. FASEB J 2011;25:1314-1322.

23 Khaidakov M, Mitra S, Kang BY, Wang X, Kadlubar S, Novelli G, Raj V, Winters M, Carter WC, Mehta JL: Oxidized LDL receptor 1 (OLR1) as a possible link between obesity, dyslipidemia and cancer. PLoS One 2011;6:e20277.

24 Lu J, Mitra S, Wang X, Khaidakov M, Mehta JL: Oxidative stress and lectin-like Ox-LDLreceptor LOX-1 in atherogenesis and tumorigenesis. Antioxid Redox Signal 2011;15: 2301-2333.

-25 Blanquicett C, Kang BY, Ritzenthaler JD, Jones DP, Hart CM: Oxidative stress modulates PPAR- $\gamma$ in vascular endothelial cells. Free Radic Biol Med 2011;48:1618-1625.

-26 Muscaritoli M, Molfino A, Bollea MR, Rossi Fanelli F: Malnutrition and wasting in renal disease. Curr Opin Clin Nutr Metab Care 2009;12:378-383.

-27 Guarnieri G, Zanetti M, Vinci P, Cattin MR, Barazzoni R: Insulin resistance in chronic uremia. J Ren Nutr 2009;19:20-24.

28 Elliott J, Mishler D, Agarwal R: Hyporesponsiveness to erythropoietin: causes and management. Adv Chronic Kidney Dis 2009;16: 94-100.

29 Locatelli F, Del Vecchio L, Pozzoni P, Andrulli S: Dialysis adequacy and response to erythropoiesis-stimulating agents: what is the evidence base? Semin Nephrol 2006;26: 269-274.

-30 Van der Steen A: Research on dialyzers with improved biocompatibility. Clin Nephrol 1986;26(suppl 1):S39-S42.

- 31 Rousseau Y, Carreno MP, Poignet JL, Kazatchkine MD, Haeffner-Cavaillon N: Dissociation between complement activation, integrin expression and neutropenia during hemodialysis. Biomaterials 1999;20:19591967.
Galli F: Vitamin E-modified dialyzers. Contrib Nephrol. Basel, Karger, 2002, vol 137, pp 95-105.

33 Del Vecchio L, Locatelli F, Carini M: What we know about oxidative stress in patients with chronic kidney disease on dialysis clinical effects, potential treatment, and prevention. Semin Dial 2011;24:56-64.

34 Galli F: Protein damage and inflammation in uraemia and dialysis patients. Nephrol Dial Transplant 2007;22(suppl 5):V20-V36.

35 Galli F, Piroddi M, Annetti C, Aisa C, Floridi E, Floridi A: Oxidative stress and reactive oxygen species. Contrib Nephrol. Basel, Karger, 2005, vol 149, pp 240-260.

- 36 Ferraro B, Galli F, Frei B, Kingdon E, Canestrari F, Rice-Evans C, Buoncristiani U, Davenport A, Moore KP: Peroxynitrite-induced oxidation of plasma lipids is enhanced in stable hemodialysis patients. Kidney Int 2003;63:2207-2213.

37 Muller C, Eisenbrand G, Gradinger M, Rath T, Albert FW, Vienken J, Singh R, Farmer PB, Stockis JP, Janzowski C: Effects of hemodialysis, dialyser type and iron infusion on oxidative stress in uremic patients. Free Radic Res 2004;38:1093-1100.

38 Ikizler TA, Morrow JD, Roberts LJ, Evanson JA, Becker B, Hakim RM, Shyr Y, Himmelfarb J: Plasma F2-isoprostane levels are elevated in chronic hemodialysis patients. Clin Nephrol 2002;58:190-197.

39 Galli F, Benedetti S, Floridi A, Canestrari F, Piroddi M, Buoncristiani E, Buoncristiani U: Glycoxidation and inflammatory markers in patients on treatment with PMMA-based protein-leaking dialyzers. Kidney Int 2005; 67:750-759.

40 Piroddi M, Palmese A, Pilolli F, Amoresano A, Pucci P, Ronco C, Galli F: Plasma nitroproteome of kidney disease patients. Amino Acids 2011;40:653-667.

41 Thornalley PJ, Rabbani N: Protein damage in diabetes and uremia - identifying hotspots of proteome damage where minimal modification is amplified to marked pathophysiological effect. Free Radic Res 2011;45: 89-100.

-42 Vanholder R, Baurmeister U, Brunet P, Cohen G, Glorieux G, Jankowski J: A bench to bedside view of uremic toxins. J Am Soc Nephrol 2008;19:863-870.

- 43 Ward RA: Protein-leaking membranes for hemodialysis: a new class of membranes in search of an application? J Am Soc Nephrol 2005; 16:2421-2430.

44 Vanholder R, Schepers E, Meert N, Lameire $\mathrm{N}$ : What is uremia? Retention versus oxidation. Blood Purif 2006;24:33-38.

$\checkmark 4$ De Smet R, Dhondt A, Eloot S, Galli F, Waterloos MA, Vanholder R: Effect of the superflux cellulose triacetate dialyser membrane on the removal of non-protein-bound and protein-bound uraemic solutes. Nephrol Dial Transplant 2007;22:2006-2012.
46 Thomas G, Jaber BL: Convective therapies for removal of middle molecular weight uremic toxins in end-stage renal disease: a review of the evidence. Semin Dial 2009;22: 610-614.

47 Rodino-Janeiro BK, Gonzalez-Peteiro M, Ucieda-Somoza R, Gonzalez-Juanatey JR, Alvarez E: Glycated albumin, a precursor of advanced glycation end-products, up-regulates NADPH oxidase and enhances oxidative stress in human endothelial cells: molecular correlate of diabetic vasculopathy. Diabetes Metab Res Rev 2011;26:550-558.

48 Bowry SK: Dialysis membranes today. Int J Artif Organs 2002;25:447-460.

49 Bergesio F, Monzani G, Manescalchi F, Boccabianca I, Passaleva A, Frizzi V: Leukocytes, eosinophils and complement function during hemodialysis with polysulphone and polymethylmethacrylate membranes: comparison with cuprophan and polyacrylonitrile. Blood Purif 1988;6:16-26.

50 Canestrari F, Galli F, Giorgini A, Albertini MC, Galiotta P, Pascucci M, Bossu M: Erythrocyte redox state in uremic anemia: effects of hemodialysis and relevance of glutathione metabolism. Acta Haematol 1994;91:187193.

51 Boaz M, Smetana S, Weinstein T, Matas Z, Gafter U, Iaina A, Knecht A, Weissgarten Y, Brunner D, Fainaru M, Green MS: Secondary prevention with antioxidants of cardiovascular disease in end-stage renal disease (SPACE): randomised placebo-controlled trial. Lancet 2000;356:1213-1218.

52 Evans HM, Bishop KS: On the existence of a hitherto unrecognized dietary factor essential for reproduction. Science 1922;56:650651.

53 Mene-Saffrane L, DellaPenna D: Biosynthesis, regulation and functions of tocochromanols in plants. Plant Physiol Biochem 2010;48:301-309.

54 Galli F, Azzi A: Present trends in vitamin E research. Biofactors 2010;36:33-42.

55 Weiser H, Vecchi M: Stereoisomers of $\alpha$ tocopheryl acetate. II. Biopotencies of all eight stereoisomers, individually or in mixtures, as determined by rat resorption-gestation tests. Int J Vitam Nutr Res 1982;52: 351-370.

56 Viola V, Pilolli F, Piroddi M, Pierpaoli E, Orlando F, Provinciali M, Betti M, Mazzini F, Galli F: Why tocotrienols work better: insights into the in vitro anti-cancer mechanism of vitamin E. Genes Nutr 2012;7:2941.

57 Zingg JM: Molecular and cellular activities of vitamin E analogues. Mini Rev Med Chem 2007;7:543-558

58 Constantinou C, Hyatt JA, Vraka PS, Papas A, Papas KA, Neophytou C, Hadjivassiliou V, Constantinou AI: Induction of caspaseindependent programmed cell death by vitamin E natural homologs and synthetic derivatives. Nutr Cancer 2009;61:864-874. 
59 Muller L, Theile K, Bohm V: In vitro antioxidant activity of tocopherols and tocotrienols and comparison of vitamin E concentration and lipophilic antioxidant capacity in human plasma. Mol Nutr Food Res 2010; 54:731-742.

60 Galli F, Mazzini F, Bamonti L, Gille L, Bohmdorfer S, Piroddi M, Netscher T, Kelly FJ, Rosenau T: Tocotrienamines and tocopheramines: reactions with radicals and metal ions. Bioorg Med Chem 2011.

61 Packer L, Weber SU, Rimbach G: Molecular aspects of $\alpha$-tocotrienol antioxidant action and cell signalling. J Nutr 2001;131:369S373 S.

-62 Thomas SR, Neuzil J, Mohr D, Stocker R: Coantioxidants make $\alpha$-tocopherol an efficient antioxidant for low-density lipoprotein. Am J Clin Nutr 1995;62:1357S-1364S.

63 Liebler DC, Burr JA, Ham AJ: Gas chromatography-mass spectrometry analysis of vitamin E and its oxidation products. Methods Enzymol 1999;299:309-318.

64 Floridi A, Piroddi M, Pilolli F, Matsumoto Y, Aritomi M, Galli F: Analysis method and characterization of the antioxidant capacity of vitamin E-interactive polysulfone hemodialyzers. Acta Biomater 2009;5:2974-2982.

-65 Sasaki M, Hosoya N, Saruhashi M: Vitamin E-modified cellulose membrane. Artif Organs 2000;24:779-789.

-66 Sosa MA, Balk EM, Lau J, Liangos O, Balakrishnan VS, Madias NE, Pereira BJ, Jaber BL: A systematic review of the effect of the Excebrane dialyser on biomarkers of lipid peroxidation. Nephrol Dial Transplant 2006; 21:2825-2833.

-67 Usberti M, Gerardi G, Bufano G, Tira P, Micheli A, Albertini A, Floridi A, Di Lorenzo D, Galli F: Effects of erythropoietin and vitamin E-modified membrane on plasma oxidative stress markers and anemia of hemodialyzed patients. Am J Kidney Dis 2002;40: 590-599.

68 Kobayashi S, Moriya H, Aso K, Ohtake T: Vitamin e-bonded hemodialyzer improves atherosclerosis associated with a rheological improvement of circulating red blood cells. Kidney Int 2003;63:1881-1887.

69 Nakatan T, Takemoto Y, Tsuchida AK: The effect of vitamin E-bonded dialyzer membrane on red blood cell survival in hemodialyzed patients. Artif Organs 2003;27:214217.

-70 Cruz DN, De Cal M, Garzotto F, Brendolan A, Nalesso D, Corradi V, Ronco C: Effect of vitamin E-coated dialysis membranes on anemia in patients with chronic kidney disease: an Italian multicenter study. Int J Artif Organs 2008;31:545-552.

-71 Cruz DN, de Cal M, Ronco C: Oxidative stress and anemia in chronic hemodialysis: the promise of bioreactive membranes. Contrib Nephrol. Basel, Karger, 2008, vol 161, pp 89-98.
72 Tarng DC, Huang TP, Liu TY, Chen HW, Sung YJ, Wei YH: Effect of vitamin E-bonded membrane on the 8-hydroxy-2'-deoxyguanosine level in leukocyte DNA of hemodialysis patients. Kidney Int 2000;58:790799.

73 Miyazaki $H$, Matsuoka $H$, Itabe $H$, Usui M, Ueda S, Okuda S, Imaizumi T: Hemodialysis impairs endothelial function via oxidative stress: effects of vitamin E-coated dialyzer. Circulation 2000;101:1002-1006.

74 Clermont G, Lecour S, Cabanne JF, Motte G, Guilland JC, Chevet D, Rochette L: Vitamin E-coated dialyzer reduces oxidative stress in hemodialysis patients. Free Radic Biol Med 2001;31:233-241.

75 Westhuyzen J, Saltissi D, Stanbury V: Oxidative stress and erythrocyte integrity in endstage renal failure patients hemodialysed using a vitamin E-modified membrane. Ann Clin Lab Sci 2003;33:3-10.

76 Bufano G, Usberti M, Mandolfo S, Malberti F, Piroddi M, Galli F: Von Willebrand factor and autoantibodies against oxidized LDL in hemodialysis patients treated with vitamin E-modified dialyzers. Int J Artif Organs 2004;27:214-221.

77 Mydlik M, Derzsiova K, Racz O, Sipulova A, Lovasova E, Molcanyiova A, Petrovicova J: Vitamin E-coated dialyzer and antioxidant defense parameters: a three-month study. Semin Nephrol 2004;24:525-531.

78 Calò LA, Naso A, Pagnin E, Davis PA, Castoro $\mathrm{M}$, Corradin $\mathrm{R}$, Riegler $\mathrm{P}$, Cascone $\mathrm{C}$, Huber W, Piccoli A: Vitamin E-coated dialyzers reduce oxidative stress-related proteins and markers in hemodialysis - a molecular biological approach. Clin Nephrol 2004;62:355-361.

79 Odetti P, Traverso N, Monacelli F, Menini S, Vazzana J, Tasso B, Pronzato MA, Robaudo C, Deferrari G: Vitamin E-coated filter decreases levels of free 4-hydroxyl-2-nonenal during haemodialysis sessions. Free Radic Res 2006;40:207-212.

80 Washio K, Inagaki M, Tsuji M, Morio Y, Gotoh H, Gotoh Y, Oguchi K: Correlation between leukocyte membrane lipid peroxidation and expression of $\mathrm{Cu} / \mathrm{Zn}$ superoxide dismutase MRNA in hemodialysis patients. Blood Purif 2011;33:59-65.

81 Akiyama S, Inagaki M, Tsuji M, Gotoh H, Gotoh T, Washio K, Gotoh Y, Oguchi K: Comparison of effect of vitamin E-coated dialyzer and oral vitamin $\mathrm{E}$ on hemodialysisinduced $\mathrm{Cu} / \mathrm{Zn}$ superoxide dismutase. Am J Nephrol 2005;25:500-506.

82 Omata M, Higuchi C, Demura R, Sanaka T, Nihei H: Reduction of neutrophil activation by vitamin E-modified dialyzer membranes. Nephron 2000;85:221-231.

83 Betjes MG, Hoekstra FM, Klepper M, Postma SM, Vaessen LM: Vitamin E-coated dialyzer membranes downregulate expression of monocyte adhesion and co-stimulatory molecules. Blood Purif 2004;22:510-517.
84 Zaluska WT, Ksiazek A, Roliski J: Effect of vitamin E-modified cellulose membrane on human lymphocyte, monocyte, and granulocyte CD11b/CD18 adhesion molecule expression during hemodialysis. ASAIO J 2001;47:619-622.

85 Pertosa G, Grandaliano G, Soccio M, Martino C, Gesualdo L, Schena FP: Vitamin Emodified filters modulate Jun $\mathrm{N}$-terminal kinase activation in peripheral blood mononuclear cells. Kidney Int 2002;62:602-610.

86 Tsuruoka S, Kawaguchi A, Nishiki K, Hayasaka T, Fukushima C, Sugimoto K, Saito T, Fujimura A: Vitamin E-bonded hemodialyzer improves neutrophil function and oxidative stress in patients with end-stage renal failure. Am J Kidney Dis 2002;39:127-133.

87 Libetta C, Zucchi M, Gori E, Sepe V, Galli F, Meloni F, Milanesi F, Dal Canton A: Vitamin E-loaded dialyzer resets PBMC-operated cytokine network in dialysis patients. Kidney Int 2004;65:1473-1481.

88 Kojima K, Oda K, Homma H, Takahashi K, Kanda Y, Inokami T, Uchida S: Effect of vitamin E-bonded dialyzer on eosinophilia in haemodialysis patients. Nephrol Dial Transplant 2005;20:1932-1935.

89 Huraib S, Tanimu D, Shaheen F, Hejaili F, Giles C, Pagayon V: Effect of vitamin E-modified dialysers on dialyser clotting, erythropoietin and heparin dosage: a comparative crossover study. Am J Nephrol 2000;20:364368.

90 Yoshida K, Kitauchi T, Kimura S, Yoneda T, Uemura H, Ozono S, Hirao Y: Serum neopterin monitoring and vitamin E-modified, regenerated hemodialyzer membrane influence on biocompatibility. Artif Organs 2002;26:54-57.

91 Baragetti I, Furiani S, Vettoretti S, Raselli S, Maggi FM, Galli F, Catapano AL, Buccianti $\mathrm{G}$ : Role of vitamin E-coated membrane in reducing advanced glycation end products in hemodialysis patients: a pilot study. Blood Purif 2006;24:369-376.

92 Kirmizis D, Papagianni A, Belechri AM, Memmos D: Effects of vitamin E-coated membrane dialyser on markers of oxidative stress and inflammation in patients on chronic haemodialysis. Nephrol Dial Transplant 2011;26:2296-2301.

93 Takouli L, Hadjiyannakos D, Metaxaki P, Sideris V, Filiopoulos V, Anogiati A, Vlassopoulos D: Vitamin E-coated cellulose acetate dialysis membrane: long-term effect on inflammation and oxidative stress. Ren Fail 2010;32:287-293.

94 Washio K, Inagaki M, Tsuji M, Morio Y, Akiyama S, Gotoh H, Gotoh T, Gotoh Y, Oguchi $\mathrm{K}$ : Oral vitamin C supplementation in hemodialysis patients and its effect on the plasma level of oxidized ascorbic acid and $\mathrm{Cu} / \mathrm{Zn}$ superoxide dismutase, an oxidative stress marker. Nephron Clin Pract 2008;109:C49C54. 


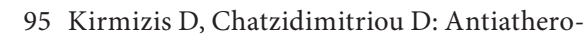
genic effects of vitamin E: the search for the Holy Grail. Vasc Health Risk Manag 2009;5: 767-774.

96 Galli F: On the antiatherogenic effects of vitamin E: the search for the Holy Grail. Vasc Health Risk Manag 2010;6:69-71.

97 Andrulli S, Di Filippo S, Manzoni C, Stefanelli L, Floridi A, Galli F, Locatelli F: Effect of synthetic vitamin E-bonded membrane on responsiveness to erythropoiesis-stimulating agents in hemodialysis patients: a pilot study. Nephron Clin Pract 2010;115:C82C89.

98 Panichi V, Rosati A, Paoletti S, Ferrandello P, Migliori M, Beati S, Bernabini G, Daini R, Casani A, Angelini D, Parrini M, Rossi A, Petrone I, Barsotti G, Donadio C, Donati G, Grazi G, Manca Rizza G, Garosi G, Sansoni E, Braccagni B, Sidoti A, Boracelli D, Biagioli M, Moriconi L, Finato V, Mannarino A, Grimaldi C, Pansa F, Imperiali P, Mura C, Bianchi S, Bigazzi R: A vitamin E-coated polysulfone membrane reduces serum levels of inflammatory markers and resistance to erythropoietin-stimulating agents in hemodialysis patients: results of a randomized cross-over multicenter trial. Blood Purif 2011;32:7-14.
99 Mandolfo S, Corradi B, Bucci R, Farina M, Pilolli F, Galli F: Evaluation of the impact of a new synthetic vitamin E-bonded membrane on anemia and rhuEPO requirement in ESRD patients with central venous catheters: a pilot study. Int Urol Nephrol 2012 (E-pub ahead of print).

100 Calò LA, Naso A, D’Angelo A, Pagnin E, Zanardo M, Puato M, Rebeschini M, Landini S, Feriani M, Perego A, Malagoli A, Zagatti R, Calzavara P, Cascone C, Davis PA: Molecular biology-based assessment of vitamin E-coated dialyzer effects on oxidative stress, inflammation, and vascular remodeling. Artif Organs 2011;35:E33-E39.

01 Morimoto H, Nakao K, Fukuoka K, Sarai A, Yano A, Kihara T, Fukuda S, Wada J, Makino $\mathrm{H}$ : Long-term use of vitamin E-coated polysulfone membrane reduces oxidative stress markers in haemodialysis patients. Nephrol Dial Transplant 2005;20:27752782.
02 Morena M, Gausson V, Mothu N, Bouchet JL, Chanas M, Grandvuillemin M, Robert A, Vela C, Canaud B, Cristol JP, Olmer M: Regional variations of low-density lipoprotein oxidizability in hemodialysis patients may explain discrepancies in interventional therapy on oxidative profile. Blood Purif 2008;26:300-310.

103 Matsumura M, Sasaki H, Sekizuka K, Sano H, Ogawa K, Shimizu C, Yoshida H, Kobayashi S, Koremoto M, Aritomi M, Ueki K: Improved management of intradialytic hypotension using vitamin E-bonded polysulfone membrane dialyzer. Int J Artif Organs 2010;33:147-153.

104 Aoun B, Janssen-Lozinska Y, Ulinski T: Effect of vitamin E-coated dialyzers on anticoagulation requirement in hemodialyzed children. Saudi J Kidney Dis Transpl 2010; 21:466-470.

05 Mazzini F, Betti M, Canonico B, Netscher T, Luchetti F, Papa S, Galli F: Anticancer activity of vitamin E-derived compounds in murine C6 glioma cells. ChemMedChem 2010;5:540-543. 